\title{
A variabilidade inter e intra-sujeito no desenvolvimento fonológico de crianças gêmeas e não gêmeas
}

\author{
Maria de Fátima de Almeida Baia \\ Universidade Estadual do Sudoeste da Bahia (UESB), Vitória da Conquista, Bahia, Brasil \\ mariadefatimabaia@uesb.edu.br
}

DOI: http://dx.doi.org/10.21165/el.v46i2.1748

\begin{abstract}
Resumo
Este é um estudo inicial e qualitativo a respeito da manifestação dos templates, isto é, rotinas articulatórias usadas para expansão do léxico, no desenvolvimento fonológico de uma criança não gêmea $(\mathrm{L})$ e de duas crianças gêmeas $\left(\mathrm{M}_{\mathrm{G}} \& \mathrm{~B}_{\mathrm{G}}\right)$ de 1;0 - 2;0 (total de 24 sessões). Após análise dos dados, verificou-se a manifestação de diferentes templates no desenvolvimento fonológico de cada criança gêmea e não gêmea. Surpreendentemente, as crianças gêmeas não mostraram o uso de uma mesma rotina articulatória, embora o mesmo contexto e ambiente estivessem sendo compartilhados pelas duas.
\end{abstract}

Palavras-chave: templates; gêmeos; desenvolvimento fonológico.

Inter and Intra-variability in the phonological development of singletons and twins

\begin{abstract}
This is an initial and qualitative study on the manifestation of templates, this is an early articulatory routines used for lexicon expansion, in the phonological development of a singleton $(\mathrm{L})$ and twins $\left(\mathrm{M}_{\mathrm{T}} \& \mathrm{~B}_{\mathrm{T}}\right)$ aged $1-2$ years (24 sessions). In the analysis, different templates were observed in L and M \& B speech. Surprisingly, the twins did not show the use of the same articulatory routine, although the same context and environment were being shared by both.
\end{abstract}

Keywords: templates; twins; phonological development.

\section{Introdução}

Este estudo analisa a emergência de templates e foca a variabilidade no desenvolvimento fonológico de crianças adquirindo o português da variedade baiana de Vitória da Conquista. A discussão baseia-se na perspectiva emergentista dos Sistemas Adaptativos Complexos (THELEN; SMITH, 1994; KELSO, 1995), que prevê mudança, gradiência, instabilidade, variabilidade e não linearidade, ao longo do desenvolvimento. A proposta fonológica assumida na abordagem dos dados infantis é a da WholeWord/Templatic Phonology (WATERSON, 1971; VIHMAN; CROFT, 2007).

A Teoria dos Sistemas Adaptativos Complexos entende o desenvolvimento da linguagem como um fenômeno de evolução, no qual as representações não são estáticas e podem ser graduais. Ele também é entendido como um fenômeno comportamental e emergente, sendo a linguagem uma habilidade cognitiva que depende de capacidades motoras e auditivas e, principalmente, do estímulo do ambiente.

Uma das características fundamentais da abordagem da complexidade é a tentativa de explicar o que é o caótico, o que aparentemente é desviante no percurso, por meio de uma perspectiva emergentista. É sabido que uma teoria de desenvolvimento 
precisa oferecer explicação acerca do surgimento do novo, das regularidades ao longo do trajeto e da precisão de determinados momentos de mudança que tendem a acontecer da mesma maneira em diferentes indivíduos. Porém, o que a perspectiva da complexidade tende a enfatizar é que não se pode ignorar a diversidade, variedade, flexibilidade e a assincronia que tendem a ocorrer no desenvolvimento.

Nos estudos de desenvolvimento fonológico (VIHMAN; CROFT, 2007), essa perspectiva de caráter dinâmico contribui com a sua ênfase no papel da variabilidade no avanço do desenvolvimento, no papel da auto-organização para a maturação do sistema, isto é, seu papel na formação de padrões, e na interconexão entre percepção, ação e aprendizagem. Em suma, a linguagem, nessa perspectiva, é entendida como uma habilidade cognitiva que depende de outros aspectos cognitivos e mecanismos como atenção, memória, capacidades motoras e auditivas. Ao assumir tal perspectiva, o presente estudo não parte de uma dualidade isolante entre mente - cérebro, ou seja, são considerados elementos de nível mental e neural em conjunto, e enfatiza o papel da interação. Ademais, há um enfoque nos aspectos de variabilidade no desenvolvimento da linguagem a fim de se verificar (des)encontros de rotinas articulatórias iniciais de crianças gêmeas e não gêmeas.

\section{A manifestação dos templates nos dados iniciais}

Como apresentado na seção anterior, o sistema fonológico, neste estudo, é entendido como um sistema dinâmico, assim como o sistema linguístico em geral.

Dentro dessa proposta dinâmica, estudos sobre a emergência da fonologia nos dados das crianças propõem a existência de templates no desenvolvimento (VIHMAN; CROFT, 2007), os quais são explicados como modelos sistemáticos temporários que facilitam a expansão do léxico. Tratam-se de produções abstratas/fonéticas que integram a palavra ou frase-alvo e padrões vocálicos. Templates consistem em uma ou mais estruturas sistemáticas que envolvem posições prosódicas que tendem a ser preenchidas com um repertório segmental limitado. Podem ser entendidos como padrões/rotinas que emergem a partir da forma-alvo e que são frequentemente usados pela criança com base nas formas fonológicas já adquiridas. As palavras reduplicadas frequentes nos dados infantis do português brasileiro (PB) podem, por exemplo, ser resultado de uma manifestação de template. A harmonia consonantal, segundo Vihman e Velleman (2000), é a manifestação mais recorrente de um template na aquisição de diferentes línguas (catalão, inglês, estoniano), o que reflete a dificuldade em mudar modo e ponto de articulação em uma mesma palavra.

De acordo com Vihman e Velleman (2000), templates podem ser classificados como selecionados ou adaptados: o primeiro refere-se às tentativas da criança que estão próximas do alvo adulto, ou seja, derivam diretamente do alvo; o segundo refere-se às adaptações que a criança faz do alvo para satisfazer o padrão presente na sua fala, algum processo fonológico que mude a palavra como um todo (apagamento, assimilação, metátese, etc.), de uma maneira sistemática. ${ }^{1}$

\footnotetext{
${ }^{1} \mathrm{O}$ termo template não pode ser igualado ao uso e significado assumido pelos estudos de aquisição de acento (GERKEN, 1994; SANTOS, 2007; BAIA, 2010), por não se referir apenas à estrutura prosódica. Por exemplo, quando Santos afirma que o modelo prosódico inicial do português é o iambo, a autora está se referindo ao pé, uma unidade prosódica, que não traz informação específica sobre o tipo de segmento que preenche a estrutura. Além disso, pé fonológico não é uma rotina instável, mas uma unidade fonológica.
} 
Por mais que os templates apresentem as mesmas origens na sua manifestação em diferentes crianças adquirindo diferentes línguas, o mesmo template não se manifesta, obrigatoriamente, na fala de todas as crianças. Essa variabilidade encontrada na produção de diferentes crianças não deriva, segundo Vihman e Velleman (2000), do input adulto, mas do "filtro" individual que cada criança carrega no processo de aquisição lexical.

Embora haja variabilidade, de acordo com os estudos citados, é possível levantar algumas generalizações das características segmentais e prosódicas que tendem a estar presentes na manifestação dos templates:

a. Os templates refletem um número limitado de estruturas silábicas que nunca excedem dois núcleos silábicos: CV, VC, CVC, CVCV, CVCVC.

b. Clusters consonantais estão geralmente ausentes.

c. Os templates são construídos a partir de um inventário segmental limitado, geralmente um subgrupo oriundo da língua-alvo. O subgrupo tende a variar de criança para criança e tem influência da continuidade articulatória do balbucio e das primeiras palavras.

d. A variação consonantal no item lexical é restrita ao modo ou ponto de articulação, não podendo ser nos dois ao mesmo tempo.

e. Há casos, embora raros, que caracterizam um template por meio de uma sequência consonantal específica.

$\mathrm{Na}$ literatura brasileira, os estudos sobre templates são recentes e poucos; além dos estudos da presente autora, há o estudo de Oliveira-Guimarães (2012) sobre aquisição de alofones. A autora verifica a manifestação de templates ao analisar dados de duas crianças (1;9 - 1;11 anos) adquirindo o português brasileiro (PB) e observa o predomínio de sistematização com dissílabos e reduplicação de sílaba:

\begin{tabular}{lll} 
Produção infantil & & Alvo \\
$(01)$ [be'be] & \multicolumn{2}{c}{ Gabriel } \\
$(02)$ [du'du] & Pedro \\
(03) [be'be] & bebê \\
$(04)$ [vo'vo] & vovó
\end{tabular}

(OLIVEIRA-GUIMARÃES, 2012)

A autora encontra, nos dados do $\mathrm{PB}$, evidências de template caracterizado por assimilação regressiva como em ['papu] 'sapo' e ['pэpo] 'copo'.

Interessantemente, muitas dessas produções iniciais, oriundas da manifestação de um template, têm sido reportadas na literatura como criação lexical e/ou causado reflexões a respeito de como categorizar tais produções infantis. Gerken (2009) chama a atenção para o que se considera protopalavra e que não pode ser confundido com palavra inicial. Protopalavra, segundo a autora, é a produção que se distancia do alvo, geralmente composta por uma ou duas sílabas, e é usada em determinados contextos pela criança para

$\mathrm{O}$ uso que Vihman e colegas fazem do termo refere-se à manifestação de um template por meio de um processo de reduplicação, ou de metátese, ou de apagamento, ou de repetição de determinado tipo de segmento sem relação com o alvo, ou qualquer outro tipo de padrão na produção das primeiras palavras. Templates carregam informações prosódicas e/ou segmentais e são caracterizados pela sua manifestação nos processos que se repetem de maneira sistemática na produção das palavras. Logo, não é qualquer processo ou ocorrência que será caracterizado como template, mas sim o que é sistemático e serve como meio de expansão do léxico. 
chamar a atenção do adulto, como, por exemplo, [di], usado para se referir a um objeto específico (GERKEN 2009, p. 73). ${ }^{2}$ Se protopalavra é a sequência de sons que, embora carregue uma significação, não tem relação com a combinação de sons da forma-alvo, então esse tipo de produção incluiria as criações lexicais presentes no período inicial (cf. SECCO, 1994; BAIA, 2010). Além disso, para Gerken, palavra inicial é uma sequência de sons próxima à do adulto, como, por exemplo [dæ] para daddy, em que os sons presentes na forma-alvo podem ser mantidos na produção da criança ou sofrer alguns processos fonológicos da fala infantil, como a reduplicação de uma sílaba do alvo, metátese, harmonia vocálica, harmonia consonantal ou truncamento.

Logo, partindo da abordagem da autora, é complicado estabelecer o limite entre um tipo de produção e outra, atentando apenas para similaridade fônica entre a produção infantil e a alvo. Isto é, se a protopalavra se distancia da forma-alvo, mas as palavras iniciais podem também sofrer processos fonológicos que distanciam a produção infantil da forma-alvo, como estabelecer a dessemelhança que caracteriza os dois tipos? Por essa razão, outros parâmetros precisam ser levados em consideração para se certificar se o que está sendo analisado pode ser entendido como palavra ou não.

Secco (1994), baseando-se em MacWhinney (2000 [1991]) e o formato Chat de transcrição ${ }^{3}$, considera as palavras, que contém significado na fala infantil, mas que fogem da forma-alvo, "criações lexicais"" . Segundo o autor, as criações lexicais são instáveis, um tipo específico de léxico que não faz parte daquele estabelecido na comunidade linguística em que a criança está inserida. $\mathrm{O}$ autor prossegue afirmando que as criações lexicais funcionam como preenchedores de lacunas da fala da criança e que podem fugir da fonotática da língua alvo, pois encontra produções de alguns itens que não seguem o padrão fonotático da língua, como, por exemplo, [km. 'km] "quero" ou "caneta". Por outro lado, Baia (2010) considera que as criações lexicais podem refletir possibilidades sonoras, que não fogem da fonotática da língua alvo, testadas pelas crianças. Nos dados levantados na pesquisa da autora, não houve caso que violasse a fonotática do português, nem mesmo nos anteriores a 1;8 anos, faixa etária analisada por Secco (1994). Baia (2010) também, ao contrário de Secco (1994), não considera as onomatopeias como criações lexicais porque elas são estáveis, isto é, "au-au" e "miau" parecem conservar-se ao longo da aquisição e até após esse período. Todavia, Secco (1994) e Baia (2010), assim como MacWhinney (2000), vão diferenciar o que é chamado de criação lexical, nas suas análises e @c no formato Chat de transcrição, de neologismo.

Dessa maneira, seguindo o raciocínio de Secco (1994) e Gerken (2009), as criações lexicais, isto é, qualquer tipo de distorção de palavra no vocabulário infantil, seriam uma protopalavra, o que é repensado no presente estudo.

O que Gerken (2009) considera protopalavra pode ser considerado palavra inicial na perspectiva da Whole-Word (Templatic) Phonology (VIHMAN; CROFT, 2007) se houver evidência de que tais produções são fruto da manifestação de um template

\footnotetext{
${ }^{2}$ A autora não diz qual seria o objeto.

${ }^{3}$ No manual do formato Chat, essas produções são descritas da seguinte maneira: Child-invented forms: are words created by the child sometimes from other words without obvious derivational morphology. Sometimes they appear to be sound variants of other words. Sometimes their origin is obscure. (MacWHINNEY, 2000, p. 38).

${ }^{4}$ No formato Chat de transcrição child-invented form@c.
} 
predominante. Dessa maneira, no presente estudo, a classificação entre protopalavra e palavra inicial não é levada em consideração.

Neste estudo, apresentamos uma perspectiva alternativa para explicação do fenômeno das "criações lexicais" ou "protopalavra" na fala infantil. Nossa hipótese é a de que criações lexicais não são invenções na fala infantil, elas são adaptações feitas de acordo com a rotina articulatória da criança no momento. Essas produções não fogem da fonotática da língua alvo e podem ser explicadas como resultado de um template operante (VIHMAN; CROFT, 2007).

Com base no que foi apresentado até então, a hipótese deste estudo é que toda e qualquer criança apresenta uso de tais rotinas articulatórias (VIHMAN; CROFT, 2007; BAIA, 2014). No entanto, a manifestação dos templates não é, necessariamente, uniforme no desenvolvimento de crianças adquirindo uma mesma variedade linguística devido à variabilidade característica da formação de qualquer sistema complexo (THELEN; SMITH, 1994). Investigamos o uso de diferentes rotinas articulatórias no desenvolvimento fonológico de crianças gêmeas.

\section{Desenvolvimento fonológico de gêmeos}

São poucos os estudos sobre o desenvolvimento fonológico de crianças gêmeas e naqueles, até então encontrados na revisão teórica do presente estudo, observa-se falta de consenso a respeito do trajeto articulatório e fônico das crianças gêmeas. Em geral, são reportados na literatura possíveis atrasos no desenvolvimento linguístico de crianças gêmeas devido ao fato de uma criança do par ter menos fala dirigida para ela e não são excluídos fatores biológicos (SMITH, 2011). Além disso, são observadas certas tendências no desenvolvimento linguístico de gêmeos como: a) tendem a completar a sentença um do outro; b) usam menos o seu nome próprio que as demais crianças; c) uso de idioglossia/criptofasia - sistema linguístico único criado e usado entre gêmeos; d) uso de linguagem simplificada; e) ocorrência de "erros" sistemáticos que não acontecem na fala de crianças não gêmeas.

Dodd e McEvoy (1994) realizam um estudo comparativo entre a fala de crianças gêmeas e não gêmeas e defendem que o inventário e o trajeto fônico de gêmeos não diferem das outras crianças. No entanto, a fala de gêmeos, segundo os autores, apresentaria atraso em relação à fala de não gêmeos devido à percepção linguística tardia da forma alvo. Isso provavelmente seria decorrente do fato de gêmeos terem menos interação com adultos do que crianças que se desenvolvem rodeadas mais de adultos. Os autores notam também que crianças gêmeas não compartilham, necessariamente, os mesmos processos fonológicos, embora não haja discrepância no desenvolvimento fonológico delas.

No grupo de estudos que comparam o desenvolvimento fonológico de gêmeos, de um lado, Bloch (1921) observa percurso fônico idêntico no desenvolvimento fonológico de crianças gêmeas, no entanto, seu estudo enfatiza dados aspectos segmentais. De outro lado, Leonard et al. (1980) e Smith (2011) observam percursos segmentais diferenciados no desenvolvimento de crianças gêmeas, isto é, irmãos/irmãs gêmeos apresentam, segundo os autores, idiossincrasias no aspecto fônico do desenvolvimento.

No que se refere aos templates, Smith (2011), em um estudo qualitativo sobre desenvolvimento fonológico do francês por crianças gêmeas de 1 ano e 3 meses -2 anos, 
observa que o par analisado compartilha os mesmos templates ao longo do desenvolvimento, tais como rotina de reduplicação total e rotina de dissílabos com consoante nasal no ataque silábico. Todavia, não é dito no estudo se as rotinas, isto é, templates, foram usadas simultaneamente pelos irmãos numa mesma sessão ou se ocorreram de modo geral no intervalo de quase um ano investigado. Dessa maneira, os resultados ficam comprometidos, pois é esperado que os irmãos compartilhem rotinas semelhantes por estarem em um mesmo ambiente adquirindo uma mesma língua-alvo. É preciso realizar uma verificação por sessão de análise, o que está em andamento na presente pesquisa.

\section{Metodologia}

Os dados pertencem ao banco de dados do GEDEF (Grupo de Estudos de Desenvolvimento Fonológico). Todos os dados têm sido transcritos auditivamente pela autora deste trabalho com o uso do alfabeto fonético internacional (IPA) e de acordo com o sistema CHAT (CHILDES) de transcrição (MACWHINNEY, 2000) e contam com a verificação e julgamento de um foneticista. Havendo $90 \%$ de concordância entre os dois transcritores, os dados foram corretamente transcritos. A respeito dos $10 \%$ discordantes, após discussão, os transcritores devem chegar a um acordo sobre a produção.

Para este estudo, são analisados: a) dados de L, sexo feminino, não gêmea, de 1 a 2 anos; b) de $\mathrm{M}_{\mathrm{G}} \& \mathrm{~B}_{\mathrm{G}}{ }^{5}$, sexo feminino, gêmeas fraternas, de 1 a 2 anos. As três crianças nasceram e estão adquirindo a variedade baiana de Vitória da Conquista, cidade localizada no sudoeste do estado. Os dados coletados e analisados são naturalísticos, isto é, oriundos de estudo longitudinal com sessões mensais com duração de trinta minutos cada.

$\mathrm{Na}$ presente pesquisa, são analisados tokens diferentemente dos outros estudos sobre os templates que consideram types (VIHMAN; CROFT, 2007). Optou-se pela análise de tokens, pois se apenas types fossem considerados, pistas ou evidências de manifestação de templates e diferentes modelos prosódicos poderiam ser excluídos dos dados. Por exemplo, Baia (2014) apresenta as seguintes produções para a palavra "aranha" em uma mesma sessão de M. aos 1;6: [a.'bo] [a.'la.nja] [a.'la.na] [a.a.'ã.na] [a.'ã.na] [a.'i.na] [a.'a.na] [a.'i.ã] [a.'da.ja] [a.'ja.ja] [a.'ba.ja] [ma.'ja.na]. Se o critério para a escolha do type fosse frequência de ocorrência, mais de um type seria escolhido por não ter havido produção que se sobressaiu em relação às demais. Além disso, um argumento contra o uso de tokens pode ser, na verdade, a seu favor. A análise de tokens poderia apontar a manifestação de um determinado template de maneira equivocada, por considerar a mesma produção repetidas vezes. Porém, essa possibilidade não representa tanto risco quanto deixar de lado o indício de que repetições também demonstram uso sistemático de um padrão fônico. Para ser considerado um template operante em uma sessão de dados, ele deve ter se repetido em pelo menos $40 \%$ dos dados da sessão.

Foram feitas duas categorizações quanto aos dados. Primeiramente, entre o que é balbucio e palavra, seguindo os critérios de Vihman e McCune (1994), pois o estudo de dados iniciais, que parte da classificação do que pode ou não ser avaliado como palavra, exige categorização devido às tênues diferenças entre o que é palavra ou não no período inicial da fala. Por certo, o contexto, a semelhança fônica e a repetição da palavra com o

\footnotetext{
${ }_{\mathrm{G}}^{5}=$ gêmeas.
} 
uso constante de um mesmo sentido são fundamentais para a categorização, mas não são os únicos critérios considerados para a classificação dos dados inicias (balbucio e palavra). Com o intuito de categorizar os dois tipos de produção de uma maneira rigorosa, é preciso, indubitavelmente, comparar os dados entre si. No entanto, só a comparação interna não é suficiente. É preciso partir de uma categorização geral baseada em critérios que possam ser e tenham sido checados na análise de dados de diferentes crianças.

Vihman e McCune (1994), baseados na análise de dados de transição do balbucio às primeiras palavras de dez crianças entre nove meses e um ano e seis meses de idade, propõem critérios que partem de parâmetros contextuais e fônicos para determinar quando a palavra pode ser entendida como palavra fonológica. Sobre a necessidade de haver tais critérios, os autores lembram que tal preocupação de reconhecer o que seria "primeira palavra" tem acompanhado especialistas em fala infantil antes do advento da pesquisa psicolinguística contemporânea. Termos e conceitos têm sido propostos na busca por delimitação e entendimento acerca das produções iniciais; por exemplo, "holófrase", para palavra isolada que pode ser entendida, na verdade, como uma sentença (LAGUNA, 1927); e "vocábulo", termo proposto para não haver distinção equivocada entre o que seria uma palavra de acordo com o alvo ou algum outro tipo de verbalização (WERNER; KAPLAN, 1963). O que se percebe é que o ponto de vista assumido pelo pesquisador recorta e interpreta diferentemente o objeto. Vihman e McCune (1994) propõem, então, uma nova metodologia para identificação de palavra sem apresentar novos termos ou conceitos. Em vez de novas terminologias, os autores sugerem o uso de critérios fonológicos que levem em consideração o contexto de interação também.

Os critérios são aplicados antes da seleção prévia das produções que levantam dúvidas. $\mathrm{O}$ candidato à palavra que suscita dúvidas quanto à sua categorização deve apresentar aspectos fônicos próximos ao do alvo e/ou pistas de contexto específico de uso para não ser descartado de início. Após a identificação daqueles que suscitam dúvidas, os seguintes critérios são seguidos:

a) Critérios baseados no contexto: determinativo - verifica-se se a palavra ocorre em um contexto sugestivo de uma determinada palavra e não de outra; identificação materna - verifica-se por meio do estudo da interação adulto e criança, ou seja, se o adulto entende o candidato à palavra da mesma maneira que o pesquisador; uso múltiplo - verifica-se se a criança utiliza a mesma produção mais de uma vez no mesmo episódio; episódios múltiplos - verificase se a criança utiliza a mesma produção em diferentes episódios.

b) Critérios baseados no modelo de vocalização: correspondência complexa verifica-se se a produção da criança contém mais de dois segmentos da forma alvo; correspondência segmental exata - verifica-se se até um ouvido não treinado reconheceria a produção da criança como sendo idêntica à produçãoalvo; correspondência prosódica - verifica-se se as características prosódicas são as mesmas presentes na forma alvo, isto é, posição de acento, tipo e quantidade silábica.

c) Critérios baseados em outras vocalizações: tokens imitados - verifica-se se o candidato à palavra é uma produção imitada e se a criança compreende o que imita; ausência de variação - verifica-se se o candidato à palavra é repetido mais de uma vez sem variação fonética; ausência de usos inapropriados verifica-se se a produção mantém o mesmo significado em diferentes contextos. 
A correspondência fonética, segundo os autores, não é cópia idêntica dos segmentos do alvo. ${ }^{6}$ Vogais que ocupam lugares vizinhos no quadro do IPA, por exemplo, [a] sendo produzido como [ə] em "casa", não são tratadas como diferentes seguindo os critérios propostos. O critério de correspondência também é obedecido quando consoantes são produzidas com aspiração quando o alvo não o é, e sibilantes produzidas com troca de ponto de articulação.

Vihman e McCune (1994) propõem que um bom candidato será aquele que respeitar no mínimo quatro critérios. O presente estudo faz uso de todos os critérios e assume o número mínimo proposto pelos autores na análise dos dados. As produções que não satisfizerem pelo menos quatro critérios são categorizadas como balbucio.

\section{Análise piloto e discussão}

Neste primeiro momento do estudo, são apresentados resultados qualitativos e gerais sobre o desenvolvimento de $\mathrm{L}$ e $\mathrm{M}_{\mathrm{G}} \& \mathrm{G}_{\mathrm{G}}$.

O primeiro aspecto a ser considerado e explicado é o da transição do balbucio para as primeiras palavras das três crianças. Como Baia (2014) observa, há influência do balbucio na formação e emergência de templates, o que pode, segundo a autora, ser entendido como evidência de continuidade entre os dois períodos.

As três crianças apresentaram o seguinte quadro de transcrição entre balbucio (B) e palavras iniciais $(\mathrm{P})$ :

$$
\begin{aligned}
& \mathbf{L}: \quad \mathrm{B}>\mathrm{B} \approx \mathrm{P}>\mathrm{B}+\mathrm{P}->\mathrm{B}-\mathrm{P}+ \\
& \mathbf{M}_{\mathrm{G}}: \mathrm{B}+\mathrm{P}- \\
& \mathbf{B}_{\mathrm{G}}: \mathrm{B}+\mathrm{P}->\mathrm{B} \approx \mathrm{P}
\end{aligned}
$$

As três crianças, tanto as gêmeas como a não gêmea, apresentaram balbucio tardio, o que é característico da continuidade entre treino motor e produção linguística de caráter funcional. No entanto, L, ao contrário das irmãs gêmeas, produz mais palavras do que produções balbuciadas até o período etário analisado. Dessa maneira, nota-se um certo descompasso entre a transição do balbucio às palavras das crianças gêmeas e da criança não gêmea. Na comparação da transição para as primeiras palavras de $\mathrm{M}_{\mathrm{G}} \mathrm{e} \mathrm{B}_{\mathrm{G}}$, notou-se um balbucio tardio mais persistente e predominante na fala de $\mathrm{M}_{\mathrm{G}}$, o que demonstra um aspecto diferencial no percurso articulatório e fonológico das duas crianças.

Em relação aos templates, os seguintes templates se manifestaram ao longo das sessões analisadas:

$$
\begin{array}{ll}
\text { L: } & \mathrm{C}_{1} \mathrm{~V}_{1} .{ }^{\prime} \mathrm{C}_{\mathbf{1}} \mathrm{V}_{\mathbf{1}} \\
& \mathrm{C}_{\text {oclu }} \mathrm{V}_{1} .{ }^{\prime} \mathrm{C}_{\text {oclu }} \mathrm{V}_{2}
\end{array}
$$

\section{CV}

$\mathbf{M}_{\mathrm{G}}: \mathrm{CV}$

\footnotetext{
${ }^{6}$ Embora façam uso da palavra "exata" em "Correspondência Segmental Exata" nos critérios do item b, Vihman e McCune (1994) explicam não haver necessidade de cópia idêntica para verificação da correspondência fonética.
} 

$\mathrm{C}_{1} \mathrm{~V}_{1} . \mathrm{C}_{1} \mathrm{~V}_{1}$
$B_{G}: \quad C_{1} V_{1} . \mathbf{C}_{1} V_{1}$
Como pode ser observado, houve manifestação do template reduplicado $\mathbf{C}_{1} V_{1}$.' $\mathbf{C}_{1} \mathbf{V}_{1}$ no desenvolvimento fonológico das três crianças:

$\begin{array}{llll}\text { L: } & (5) & {[\beta a . ' \beta a]} & \text { Fafá } \\ & (6) & {[\text { mã.'mã }]} & \text { mãe } \\ \mathbf{M}_{\mathrm{G}}: & (7) & {[\text { pa.'pa }]} & \text { comida } \\ & (8) & {[\text { mã.'mã }]} & \text { mãe } \\ \mathbf{B}_{\mathrm{G}}: & (9) & {[\mathrm{pa} \cdot \mathrm{pa}]} & \text { comida } \\ & (10) & {[\mathrm{mu} . ' \mathrm{mu}]} & \text { Malu }\end{array}$

De um lado, segundo Lewis (1936), a reduplicação fonológica na fala infantil pode ser entendida como "pedaços de balbucio", e, segundo Fee e Ingram (1982), como "vestígios de balbucio". De outro lado, Jakobson (1972 [1941]), apesar de apresentar exemplos de palavras infantis reduplicadas no russo (tutu "escondido", dada "tio", nana "enfermeira", nuna "choro") para explicar a aquisição de diferentes vogais, não traça nenhuma relação entre a reduplicação e o balbucio. No trabalho de 1962, "Why 'mama' and "papa", Jakobson explica, retomando seu trabalho com Halle (1956), que essas palavras infantis, como outras presentes na fala da criança, são baseadas na polaridade entre a consoante ótima e a vogal ótima. Apesar de o autor reconhecer que: "na transição do balbucio para o comportamento verbal, a reduplicação pode servir como um processo compulsório, sinalizando que os sons internalizados não representam um balbucio, mas uma entidade dotada de sentido ${ }^{7 "}$ (JAKOBSON, 1962, p. 543, tradução nossa, grifo do autor), ele não observa que o fato de haver um processo que, segundo suas próprias palavras, é compulsório e não pode ser considerado balbucio - apesar de compartilhar características com o período anterior e apresentar significado - , pode trazer complicações para a sua visão a favor da descontinuidade, pois o autor, ao reconhecer semelhanças entre o processo de reduplicação e o momento de balbucio, traça uma continuidade entre eles.

Além de ser um processo fonológico comum, os dados analisados também mostram a relação entre a reduplicação fonológica e a manifestação de template. $\mathrm{O}$ seu predomínio como template nos dados iniciais ocorre, aparentemente, devido à influência das produções do balbucio.

$\mathrm{L}$, diferentemente das crianças gêmeas, manifesta ao longo do seu desenvolvimento o uso de rotina com informação segmental em $\mathrm{C}_{\text {oclu }} \mathrm{V}_{1}$. ' $\mathrm{C}_{\text {oclu }} \mathrm{V}_{2}$, com uso de consoante oclusiva em posição de ataque silábico aos $1 ; 11$ :
L:
$\begin{array}{ll}\text { (11) } & {[\text { 'to.to }]} \\ \text { (12) } & \text { ['do.te }]\end{array}$
foto
(12) ['do.te] doce

\footnotetext{
7 "At the transition from babbling to verbal behaviour, the reduplication may even serve as a compulsory process, signaling that the uttered sounds do not represent a babble, but a senseful semantic entity".
} 
É importante chamar a atenção para o aspecto efêmero dessa rotina que caracterizou a sessão de $1 ; 11$, pois a substituição sistemática de segmentos fricativos por oclusivos na fala de L. não se estendeu até a sessão de 2 anos.

$\mathrm{Na}$ comparação dos templates manifestados na fala das crianças gêmeas, $\mathrm{M}_{\mathrm{G}}$ apresentou uso sistemático de produções monossilábicas, o que não foi observado no desenvolvimento de $\mathrm{B}_{\mathrm{G}}$ :

$$
\begin{array}{rlll}
\mathbf{M}_{\mathrm{G}}: & (13) & {[\mathrm{b} \varepsilon]} & \text { Bela (nome) } \\
& (14) & {[\mathrm{mã}]} & \text { mãe }
\end{array}
$$

Tal desencontro de rotinas iniciais, observado no desenvolvimento de $\mathrm{M}_{\mathrm{G}} \& \mathrm{G}_{\mathrm{G}}$, apresenta evidências contrárias às de Smith (2011) a respeito da natureza dos templates na fala de gêmeos. Ao contrário do que é observado pela autora em dados de aquisição do francês, dados de crianças adquirindo o PB não mostram uso dos mesmos templates por gêmeos.

O quadro seguinte resume as diferenças no que se refere à transição e aos templates no desenvolvimento fonológico das crianças gêmeas e não gêmea:

\begin{tabular}{|c|c|c|c|}
\hline Criança/Idade & $L / 1-2$ anos & MG / 1-2 anos & $B_{G} / 1-2$ anos \\
\hline Transição B - P & $\mathrm{B}>\mathrm{B} \approx \mathrm{P}>\mathrm{B}+\mathrm{P}->\mathrm{B}-\mathrm{P}+$ & $\mathrm{B}+\mathrm{P}-$ & $\mathrm{B}+\mathrm{P}->\mathrm{B} \approx \mathrm{P}$ \\
\hline Templates & $\begin{array}{l}\mathbf{C}_{\mathbf{1}} \mathbf{V}_{\mathbf{1}} \cdot \mathbf{C}_{\mathbf{1}} \mathbf{V}_{\mathbf{1}} \\
\mathrm{C}_{\text {oclu }} \mathrm{V}_{1} \cdot \mathrm{C}_{\text {oclu }} \mathrm{V}_{2} \\
\mathrm{CV}\end{array}$ & $\begin{array}{l}\mathbf{C}_{\mathbf{1}} \mathbf{V}_{\mathbf{1}} . \mathbf{C}_{\mathbf{1}} \mathbf{V}_{\mathbf{1}} \\
\mathrm{CV}\end{array}$ & $\mathbf{C}_{1} \mathbf{V}_{1} . \mathbf{C}_{1} \mathbf{V}_{1}$ \\
\hline
\end{tabular}

Quadro 1. (Des)encontros no desenvolvimento fonológico das crianças gêmeas e não gêmea.

Como o quadro ilustra, observa-se variabilidade não apenas na comparação do percurso fônico de crianças gêmeas e não gêmea, como também na comparação dos dados das irmãs. Embora estejam no mesmo contexto linguístico, familiar e cultural, em contato com a mesma língua-alvo e cuidadores, os templates manifestados não foram os mesmos.

\section{Considerações finais}

Em geral, verificou-se a persistência do balbucio tardio na fala das três crianças analisadas, o que pode ser entendido como evidência de continuidade entre os dois períodos. No que se refere aos templates, todos os que foram manifestados estão de acordo com os padrões fonotáticos e prosódicos da língua-alvo, mas houve diferenças nas manifestações de cada criança. A variabilidade observada no percurso de desenvolvimento fonológico de cada criança é esperada porque o sistema fonológico é entendido como um sistema dinâmico aberto e instável. Conforme o inventário lexical da criança aumenta, mais próxima sua produção fica do que é predominante e mais frequente na língua alvo. Este estudo segue, no momento, com análise quantitativa e mapeamento de templates por sessão. 


\section{REFERÊNCIAS}

BAIA, M. F. A. O modelo prosódico inicial do português brasileiro: uma questão de metodologia? São Paulo: FFLCH/USP Produção Acadêmica Premiada, 2010.

. O papel do balbucio na formação dos templates. Estudos Linguísticos, 43(2), p. 679-695, mai./ago. 2014.

BLOCH, O. Les premiers stades du langage de l'enfant. Journal de psychologie, 1921,18, 693-712.

DODD, B.; MCEVOY, S. Twin language or phonological disorder? Journal of child language, 21, p. 273-289, 1994.

FEE, J.; INGRAM, D. Reduplication as a strategy of phonological development. Journal of child language, 9, p. 41-54, 1982.

GERKEN, L. A metrical template account of children's weak syllable omissions from multisyllabic words. Journal of child language, v. 21, p. 565-584, 1994.

. Language development. Arizona: Plural Publishing, 2009.

JAKOBSON, R. Child language, aphasia and phonological universals. Paris: Mouton, 1972 [1941].

Why "mama" and "papa"? Selected writings, v. 1: phonological studies. The Hague: Mouton, 1962. p. 538-545.

JAKOBSON, R.; HALLE, M. Fundamentals of Language. Holanda: Mouton Publishers, 1980 [1956].

KELSO, J. A. S. Dynamical Patterns: The Self-Organization of Brain and Behavior. Cambridge: MIT Press, 1995.

LAGUNA, G. Speech: its function and development. New Haven: Yale University Press, 1927.

LEONARD, L. N.; NEWHOFF, M.; MESALAM, L. Individual differences in early child phonology. Applied Psycholinguistics, 1, p. 7-30, 1980.

LEWIS, M. M. Infant Speech: a study of the beginning of language. New York: Harcourt, Brace and Company, 1936.

MACWHINNEY, B. The CHILDES project: tools for analyzing talk. v. 1: transcriptions and programs. New Jersey: Lawrence Erlbaum Associates, 2000.

OLIVEIRA-GUIMARÃES, D. Beyond early words: word template development in Brazilian Portuguese. In: VIHMAN, M.; KEREN-PORTNOY, T. (Org.). The emergence of Phonology: Whole-Word approaches, cross-linguistic evidence, Cambridge, 2012.

SANTOS, R. S. A aquisição prosódica do português brasileiro de 1 a 3 anos: padrões de palavra e processos de sândi externo. 2007. 214 f. Tese (Livre Docência em Linguística) - Faculdade de Filosofia, Letras e Ciências Humanas, Universidade de São Paulo, São Paulo, 2007.

SECCO, G. Criações lexicais em uma criança de 20 meses de idade. 1994. 203 f. Dissertação (Mestrado em Linguística) - Faculdade de Letras, Universidade Federal de Santa Catarina, Florianópolis, 1994. 
SMITH, C. E. Variation and similarity in the phonological development of French dizygotic twins: phonological bootstrapping towards segmental learning? York papers in Linguistics, v. 11, p. 74-87, 2011.

THELEN, E.; SMITH, L. B. A Dynamic Systems Approach to the Development of Cognition and Action. Cambridge, MA: MIT Press, 1994.

VIHMAN, M. M.; VELLEMAN, S. L. Phonetics and the origins of phonology. In: BURTON-ROBERTS, N.; CARR, P.; DOCHERTY, G. (Ed.). Phonological knowledge: its nature and status. Oxford: Oxford University Press, 2000. p. 305-339.

VIHMAN, M.; CROFT, W. Phonological development toward a "radical" templatic phonology. Linguistics, 45(4), p. 683-725, 2007.

WATERSON, N. Child phonology: a prosodic view. Journal of Linguistics, 7, p. 179211, 1971. Publicado novamente em: WALTERSON, N. Prosodic Phonology: The theory and its application to language acquisition and speech processing. Newcastle upon Tyne: Grevatt \& Grevatt, 1987.

WERNER, H.; KAPLAN, B. Symbols formation. Nova Iorque: Wiley and Hillsdale, NJ, 1963.

Recebido em: 16/10/2016

Aprovado em: 19/11/2016 